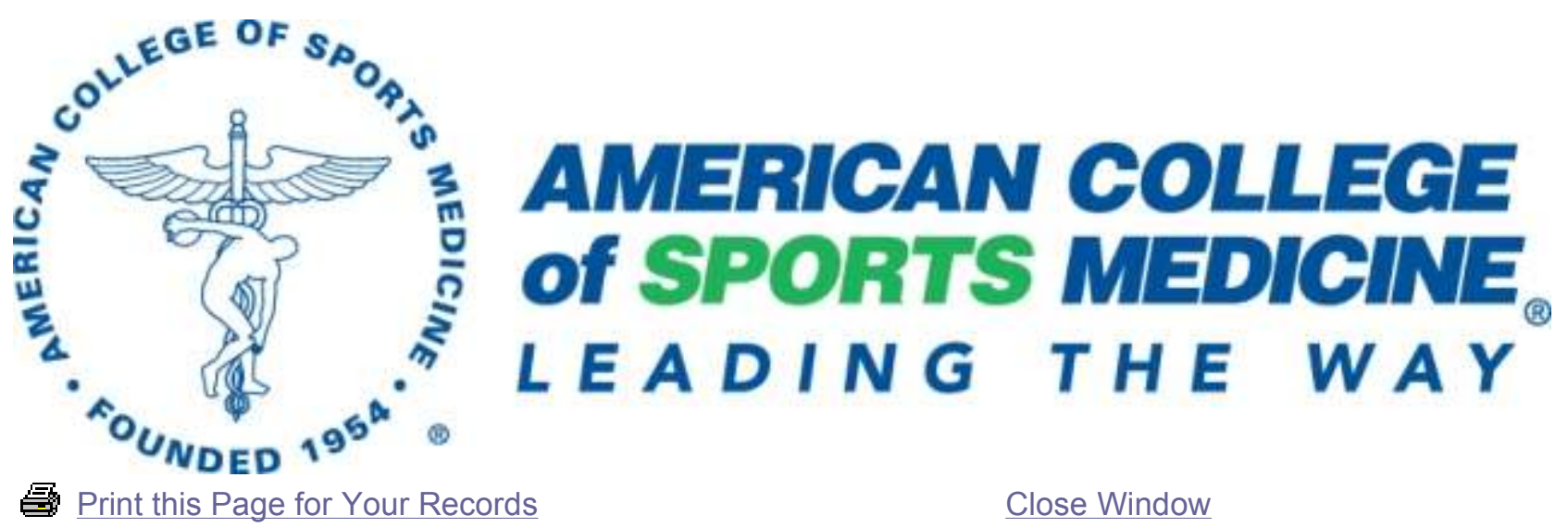

Control/Tracking Number: 10-SA-2505-ACSM

Activity: Scientific Abstract

Current Date/Time: 10/30/2009 11:38:13 AM

\title{
Effect of Carbon Graphite Foot Plate on Loading During Jumping and Landing
}

Author Block: Robin M. Queen, Alicia N. Abbey, James A. Nunley. Duke University, Durham, NC. (Sponsor: Donald Kirkendall, PhD, FACSM)

Email: robin.queen@duke.edu

Abstract:

Fifth metatarsal stress fractures have a high incidence of non-union, delayed union, and re-fracture independent of post-injury treatment. After screw fixation for the treatment of $5^{\text {th }}$ metatarsal stress fractures, many patients are being treated with a rigid carbon graphite foot plate (CFP) to decrease plantar loading.

PURPOSE: To determine if total foot, lateral midfoot (LMF) and lateral forefoot (LFF) loading (peak pressure, contact area, and force-time integral) were decreased with the use of the CFP.

METHODS: Plantar loading in 13 healthy male, collegiate, recreational athletes was evaluated using the Pedar-X in-shoe system collecting at $100 \mathrm{~Hz}$ (Novel, St. Paul, MN, USA). Exclusion criteria were any history of lower extremity injuries in the past six months, foot or ankle surgery within the past 3 years, and previous metatarsal stress fractures. Prior to data collection all subjects signed informed consent. Subjects completed 7 simulated layups in a standard running shoe with and without a CFP. In order to complete the task properly, subjects needed to jump and land on the same foot, which was the foot used for analysis. A 2x2 repeated measures ANOVA was used to compare the take-off (TO) and landing (LD) both with and without the CFP ( $\alpha=0.05)$.

RESULTS: The subjects had an average height of $1.76 \pm 0.08 \mathrm{~m}$, mass of $73.38 \pm 9.87 \mathrm{~kg}$, and age of $21.6 \pm$ 2.75 years. The total foot maximum force was significantly greater during landing when compared with the takeoff ( $p=0.012)$. No other significant differences existed between these two tasks. Independent of the task, the total foot contact area was significantly decreased in the CFP condition $(p<0.001)$, while the total foot peak pressure was increased with the CFP ( $p<0.001)$. LMF Contact area was decreased $(p=0.016)$ when wearing the CFP. No differences in the force-time integral were found in the LMF or LFF. Peak pressure was increased in the LMF $(p<0.001)$, and LFF $(p=0.002)$ when wearing the CFP.

CONCLUSIONS: In this initial study, the results indicate that the CFP is ineffective at reducing plantar loading beneath the $5^{\text {th }}$ metatarsal during jumping and landing in uninjured controls. However, CFP needs to be examined to determine its role in other sporting maneuvers and footwear in patients with a $5^{\text {th }}$ metatarsal fracture history. 
Author Disclosure Information: R.M. Queen, None.

Category (Complete): 402 sport biomechanics

Keyword (Complete): Plantar Loading ; Carbon Foot Plate ; Metatarsal Stress Fracture Unlabeled/ Investigational Products (Complete):

: No

Presentation Preference (Complete): Indifferent

Area of Interest (Complete):

Area of Interest: Applied Science

\section{Additional Info (Complete):}

*Do you authorize ACSM to record your presentation?: Yes

*Confirmation: I understand and agree to the above terms regarding AV equipment

Payment (Complete): Your credit card order has been processed on Friday 30 October 2009 at 11:35 AM.

Status: Complete

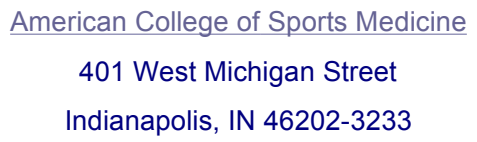

OASIS Helpdesk

Powered by OASIS, The Online Abstract Submission and Invitation System SM

(C) 1996 - 2009 Coe-Truman Technologies, Inc. All rights reserved. 